\title{
Mode Transition and Symmetry-Breaking in the Wake of a Flapping Foil
}

\author{
G. Y. He ${ }^{1, *}$, X. Zhang ${ }^{2}$, S. G. Zhang ${ }^{3}$, Q. Wang ${ }^{1}$
}

1 The School of Aircraft Engineering, Nanchang Hangkong University, Nanchang 330063, China

2 LNM, Institute of Mechanics, Chinese Academy of Sciences, Beijing 100080, China

3 The School of Aeronautic Science and Engineering, Beijing University of Aeronautics and Astronautics, Beijing 100083, China

Email: hegy509@yahoo.com.cn

\begin{abstract}
A numerical model for two-dimensional flows around a pitching foil in a viscous flow is presented. The model is numerically solved using the immersed boundary method and used to investigate the flow patterns of the foil pitching sinusoidally over a range of frequencies and amplitudes. A transition from the Karman vortex streets to the reverse Karman vortex streets are found, as the amplitudes of pitching motions increase. In the transition, the vortex streets undergo symmetry-breaking to the central lines of vortex streets. Those observations are in agreement with the previous experiment (Phys. Rev. E. 77016308 2008). Furthermore, we examine the wake of the foils pitching with different frequencies. The transition from the Karman vortex streets to the reverse Karman vortex streets is also observed. An explanation is presented to the mechanism of the transition.
\end{abstract}

\section{INTORDUCTION}

The pitching of tails is a common mode for animals to generate thrust. As a tail pitches vertically, thrust can be horizontally generated, depending on either amplitudes or frequencies of pitching motions. A simple model to understand the mechanism of pitching-generated-thrust is the Karman vortex streets (KVS) and the reverse Karman vortex streets (RKVS). At a certain range of Reynolds numbers, a KVS can be observed behind a steady foil without thrust generation. As either amplitudes or frequencies increase of pitching motions, the transition from the KVS to the RKVS can be observed and thus a thrust is generated. The present paper is devoted to numerically investigate the transition from the KVS to the RKVS.

Knoller [1] and Betz [2] were the first to observe that a flapping wing could generate thrust. Karman and Burgers [3] offered the first theoretical explanation of drag or thrust production based on the wake vortices, where the wake of the flow past bluff bodies is modeled by an infinite row of alternating vortices commonly known as von Karman Vortex Streets. In following years, many researchers investigated the RKVS in different situations, such as the wakes of fish [4] in experiment [5] and numerical simulations [6,7]. More recently, Godoy-Diana et al.[7] experimentally investigated the transition from the KVS to the RKVS in the wake of pitching foil. We will simulate the transition process using the immersed boundary method and compare the results obtained with Godoy-Diana et al's experimental results.

\section{NUMERICAL METHOD}

Two-dimensional incompressible Navier-Stokes equations are used

$$
\frac{\partial u}{\partial t}+\boldsymbol{u} \cdot \nabla \boldsymbol{u}=-\nabla p+\frac{1}{R e} \nabla^{2} \boldsymbol{u}+\boldsymbol{f}
$$


where $v$ is the kinematic viscosity. The geometry of the pitching foil is described in Figure 1, where the chord $C=$ $23 \mathrm{~mm}$ and the semicircle diameter $D=5 \mathrm{~mm}$. The foil pitches with respective to the center of the semicircle. The control parameters are the velocity $U$, the oscillation frequency $f$ and the peak to peak amplitude $A$. The main nondimension parameters are the Reynolds number Re, the pitching amplitude $A_{D}$ and the Strouhal number $S t$, defined as: $R e=U D / v, A_{D}=A / D$ and $S t=f D / U$, The pitching motion of foils is a sinusoidal function of time: $A(t)=$ $-A_{D} \sin (2 \pi f t)$.

The computational region is the one from $-3 D$ to $19 D$ in the horizontal (or streamwise) direction $x$, and from $-8 D$ to $8 D$ in the vertical direction with the origin at the centre of the semicircle. The number of uniform grids is $240 \times 120$ with $\Delta x=0.025, \Delta y=0.025$, see Figure 2 .

The boundary conditions are defined as follows: a uniform flow with $U_{0}=1$ is imposed at inlet with the normal gradient of pressure being zero, the velocity at outlet is extrapolated from interior and the pressures gradients at up and bottom boundaries are zero. A direct forcing approach of the immersed boundary method [8,9] is used for moving boundaries on a non-staggered Cartesian grid, where the forcing is computed through the volume fraction occupied by the solid and is used to mimic the non-slip boundary on the immersed surfaces [10]. A finite volume based fractional step method is used to numerically solve the N-S equations. The spatial discretization and temporal advancing schemes are both second order accurate.

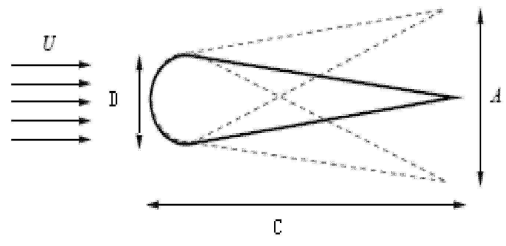

Figure 1: Schematic views of the foil

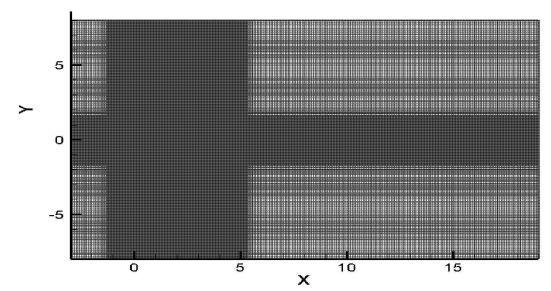

Figure 2: Grid used for the present computation

\section{NUMERICAL RESULTS}

Figure 3 shows the numerical results obtained at $R e=255$ and $S t=0.22$ : the left column is for the vorticity, the middle one for time averaged velocity component in the streamwise direction and the right one for the streamwise component of velocity in the near of wake. The first row at $A_{D}=0.36$ is the typical case of low-amplitude pitching which produces a KVS. Increasing the pitching amplitude to $A_{D}=0.71$, the vortices of alternating signs align with the symmetry line of the wake. Further increasing the amplitude to $A_{D}=1.07$, the rotation direction of vortices changes and the flows in wakes transit from the well-known KVS to the RKVS, which generate thrust on the pitching foils. It is clearly seen that the velocity profile becomes convex forward. As the amplitude increases to 1.77 , the vortex streets are deflected and asymmetric to the deflection line. Further increasing the pitching amplitude, a symmetry breaking is observed in the RKVS. All of the observations are in agreement with the reference [7]. Similarly, increasing the frequency of pitching foil, a transition from KVS to the RKVS can be also seen as shown in Figure 4. The transition is induced by large motions of pitching foils.

\section{CONCLUSION}

In this paper, we use the immersed boundary method to simulate the wake of pitching foils. We observed the transition from the KVS to the RKVS, as either amplitudes or frequencies of flaps increase. The observations are in agreement with the previous experiment in [7]. Further measurement will be carried out such as the drag coefficients, propulsion efficiency and unstable models. The latter will be used to explore the mechanism of transition of the KVS to the RKVS.

\section{Acknowledgments}

This work was supported by the Natural Science Foundation of JiangXi province under Project (2010GZC0162, 2009GZC0101). 

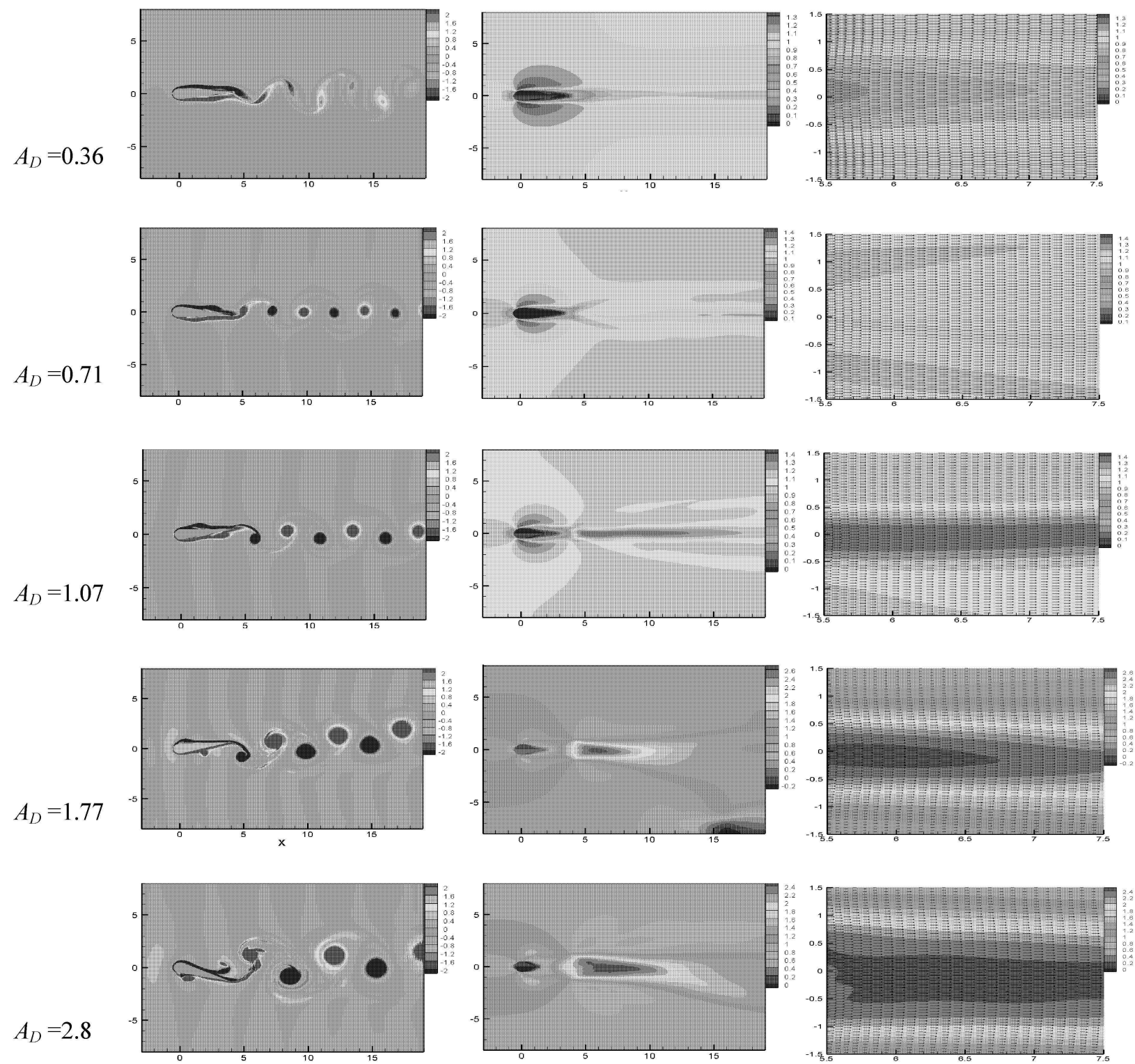

Figure 3: Instantaneous vorticity field (left column), time-averaged velocity component in stream-wise direction (middle column) and the component with direction in the near of wake (right column) for fixed $R e=255$ and $S t=0.22$, from top to bottom for $A_{D}=0.36$, $0.71,1.07,1.77,2.8$

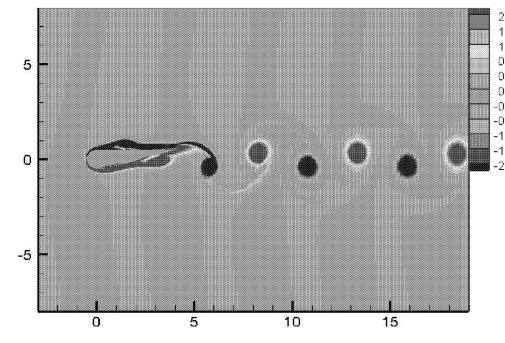

$S t=0.22$

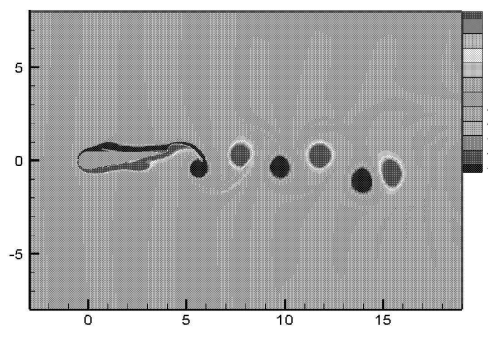

$S t=0.3$

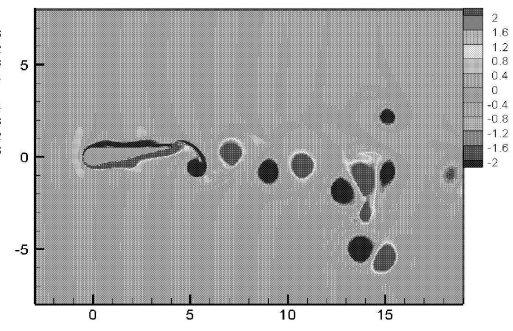

$S t=0.4$

Figure 4: Instantaneous spamwise vorticity field for fixed $R e=255$ and $A_{D}=1.07$

\section{REFERENCES}

1. Knoller R. Die Gesetze des Luftwiderstandes. Flug- und Motortechnik (Wien). 1909; 3(21): 1-7

2. Betz A. Ein Beitrag zur Erklärung des Segelfluges. Zeitschrift fur Flugtechnik und Motorluftschiffahrt. Jan. 1912; 3: 269-272 
3. von Karman T, Burgers JM. General Aerodynamic Theory Perfect Fluids. Aerodynamic Theory, W. F. Durand ed., Division E, Vol. 2, Julius-Springer, Berlin, 1943. 308

4. Drucker EG, Lauder GV. Locomotor function of the dorsal fin in teleost fishes: Experimental analysis of wake forces in sunfish. J Exp Biol, 2001; 204: 2943-2958

5. Jones KD, Dohring CM, Platzer MF. An experimental and computational investigation of the knoller-betz effect. AIAA J, 1998; 36(7): 1240-1246

6. Zhu Q, Wolfgang M, Yue J, et al. Three-dimensional flow structures and vorticity control in fish-like swimming. J Fluid Mech, 2002; 468: 1-28

7. Godoy-Diana R, Aider JL, Wesfreid JE. Transition in the wake of a flapping foil. Phys. Rev. E, 77 016308. 2008

8. Mittal R, Iaccarino G. Immersed Boundary methods. Annual Review of Fluid Mechanics, 2005; 37: 239-261

9. Kim J, Kim D, Choi H. An immersed-boundary finite-volume method for simulations of flow in complex geometries. J Comp Phys, 2001; 171: 132-150

10. Ravoux JF, Nadim A, Haj-Hariri H. An embedding method for bluff body flows: Interactions of two side-by-side cylinder wakes. Theoret. Comput. Fluid Dynamics. 2003; 16: 433-466 